\title{
Commonly Found Zoonotic Parasite Species in Dogs and Cats from a Prominent Pet Market of Dhaka, Bangladesh
}

\author{
Priyanka Barua $^{{ }^{*}}$, Sharmin Musa ${ }^{1}$, Rezwana Ahmed ${ }^{1}$ and Hamida Khanum ${ }^{1}$ \\ ${ }^{1}$ Department of Zoology, University of Dhaka, Dhaka 1000, Bangladesh.
}

Authors' contributions

This work was carried out in collaboration among all authors. Authors HK, SM and PB designed the study, wrote the protocol and performed the statistical analysis. Author PB wrote the first draft of the manuscript and managed the literature searches. Author RA performed the laboratory analyses. All authors read and approved the final manuscript.

Article Information

DOI: $10.9734 / A R R B / 2020 / v 35 i 130176$ Editor(s):

(1) Paola Angelini, Department of Applied Biology, University of Perugia, Perugia, Italy.
Reviewers: Reviewers:

(1) D. Sajitha Weeasinghe, Sri Lanka.
nolds, University of Education, Ghana. (2) Papa Kofi Amissah-Reynolds, University of Education, Ghana.
(3) Rojan Dahal, Nepal. Complete Peer review History: http://www.sdiarticle4.com/review-history/54729

Original Research Article

Received 16 December 2019

Accepted 22 February 2020

Published 14 March 2020

\section{ABSTRACT}

Background: Zoonotic parasite species are those parasites of animal origin that can be transferred to human. They possess the threat of high infection rate among both animals and human and should be monitored carefully.

Aims: The current study aimed to determine the prevalence of zoonotic parasite species in cats and dogs from a prominent pet market of Dhaka, Bangladesh.

Methodology: A total of 60 animals (30 dogs and 30 cats) were selected from different pet shops in Katabon pet market, Nilkhet, Dhaka, Bangladesh. The hosts were dogs of foreign breed; German Spitz, German Shephed (Canis lupus familiaris) and cats of local breed (Felis catus). They were age matched: Puppies/Kittens ( $\geq 6$ months -1 year), young ( $>1-2$ years) and adults ( $>2$ - 3 years) for both dogs and cats. Feces were collected and Formol Ether concentration technique was done prior to identify parasite's egg, ova and larvae by microscopy.

Results: We were able to identify 17 different parasite species of zoonotic importance in total 60 animals. 8 species were common in both animals (Taenia spp., Hymenolepis diminuta, H. nana, 
Ancylostoma spp., Ascaris lumbricoides, Capillaria spp., Toxascaris leonina and Trichuris vulpis). Apart from 8 common species, 2 more species were exclusively identified in dogs and 7 species in cats. Capillaria spp. had the highest prevalence in both dogs $(86.67 \%)$ and cats $(90 \%)$ followed by Trichuris vulpis $(83.33 \%$ in dogs, $90 \%$ in cats). Other highly prevalent parasites in dogs were $A$. lumbricoides and Toxocara canis (prevalence $76.67 \%$ for both); in cats were - T. leonina, Toxocara cati, Sarcocystis spp. and Toxoplasma spp. (prevalence 76.67\%, $73.33 \%, 60 \%$ and $60 \%$, respectively). According to the age group of hosts, in both dogs and cats, puppies or kittens and young hosts had higher prevalence of parasites compared to adults.

Conclusion: Proper training should be given to pet handlers when handling the food/feces of pets to reduce the risk of zoonotic infection and mass people should be aware about the risk of zoonotic parasite species to avoid potential health hazards.

Keywords: Zoonotic infection; cats and dogs; parasites; pets.

\section{INTRODUCTION}

Zoonotic diseases are the diseases caused by parasites which was originally found in animals and later transferred to human. This is a cause of global concern as infections in human can be established due to close contact with either domesticated or wild animals [1]. The grave consequence of zoonotic infection has been reported from ancient period (rabies) [2] as well as the recent outbreak of 2019-novel coronavirus (2019-nCoV) [3]. Although most of the cases of huge death toll results from virus related zoonotic infections, it is important to note that other parasitic infection of zoonotic importance can cause serious health issues. This includes various protozoans, cestode, nematodes of zoonotic importance and they can spread through contamination of food with excreta of animals, close contact with domesticated animals etc. [4].

Dogs (Canis lupus) and cats (Felis catus) are both from order Carnivora as they were predators in ancient time. When human habituated themselves with domestication, dogs became the first animal to be tamed [5] and later cats were also domesticated. In modern world, they became pets or animals having close emotional bond with the master as they no longer serve for their initial purpose of domestication (e.g. helping in hunting, protection from predators or rodents etc.). Instead, they have been considered as a member of the family in nuclear families where pets have deep emotional bonds with their owners [6].

Several studies have reported the prevalence of parasites of zoonotic importance in dogs and cats. Trichuris spp., Toxocara spp., Dipylidium caninum and Ancylostomatidae, Taeniidae were observed in a study from Portugal [7] in dog feces. In cats of China, the commonly found gastrointestinal parasites were Toxocara cati, Isospora felis, I. rivolta, Paragonimus, Toxoplasma, Trichuris, Sarcocystis, Trematode etc. [8]. In Europe, Toxoplasmosis, leishmaniosis, giardiosis, echinococcosis, dirofilariosis and toxocariosis were commonly found zoonotic diseases [9].

A previous study [10] in Bangladesh reported the prevalence of zoonotic parasites in dogs and cats in 2011. The dog parasites included Diphyllobothrium latum, Dipylidium caninum, Echinococcus granulosus, Ancylostoma caninum, Gnathostoma spinigerum, Toxocara canis and Trichuris vulpis while cats had Ancylostoma tubaeforme, Dirofilaria immitis, $T$. cati, Toxoplasma gondii and Paragonimus westermani. In Bangladesh, people in rural areas often have pets but currently, pets are also being taken in by urban families. As the close contact with animals happen in limited living space, risk of zoonotic diseases is much higher in urban areas. But there is scarcity of studies relating to the zoonotic parasite infestation in dogs and cats in urban areas of Bangladesh.

The aim of this study was to determine the parasite prevalence of the important parasite species of a prominent pet market of Bangladeshi capital, Dhaka city (Katabon pet market, Nilkhet). A previous report from this study [11] reported the association of dog parasites with the risk of zoonotic infection, this article reports the commonly found zoonotic parasites in both dogs and cats.

\section{MATERIALS AND METHODS}

A total of 60 animals ( 30 dogs and 30 cats) were selected for the current study from different pet shops in Katabon pet market, Nilkhet, Dhaka, 


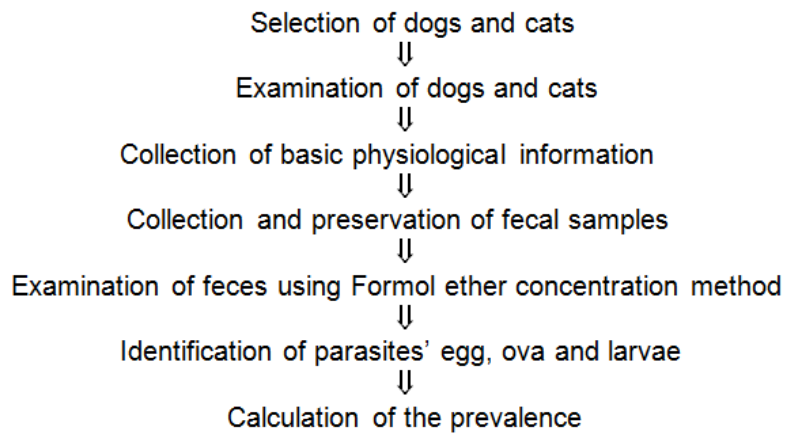

\section{Fig. 1. A flow chart showing the experimental design}

Bangladesh. The hosts were dogs of foreign breed; German Spitz, German Shephed (Canis lupus familiaris) and cats of local breed (Felis catus). They were age matched with 7 Puppies/ Kittens ( $\geq 6$ months- 1 year), 12 young (>1- 2 years) and 11 adults (>2 - 3 years) for both dogs and cats. Approximately 20-25 g of feces were collected and Formol ether concentration method [12] was used prior to microscopy to identify parasite's egg, ova and larvae (Fig. 1). Laboratory analyses was performed in the Parasitology Laboratory of Department of Zoology, University of Dhaka, Bangladesh.

\subsection{Experimental Design}

Approximately 20-25 g of feces were collected and Formol ether concentration method [12] was used prior to microscopy to identify parasite's egg, ova and larvae (Fig. 1). Laboratory analyses was performed in the Parasitology Laboratory of Department of Zoology, University of Dhaka, Bangladesh.

\section{RESULTS}

\subsection{Occurrence of Zoonotic Parasite Species in Dogs and Cats}

In the current study, 30 dogs and 30 cats were selected to report the prevalence of commonly found zoonotic parasites. In dogs, 10 species and in cats, 15 species of parasites of zoonotic importance were found. Among them, 2 were protozoan (Sarcocystis spp., Toxoplasma spp.), 4 were cestode (Taenia spp., Hymenolepis diminuta, $H$. nana, Diphyllobothrium latum), 2 were trematode (Heterophyes spp., Opisthorchis spp.), 8 were nematode (Ancylostoma spp., Ascaris lumbricoides, Capillaria spp., Gnathostoma spp., Toxascaris leonina, Toxocara canis, T. cati, Trichuris vulpis) and 1 was Pentastomid (Linguatula serrata).

Among all this parasites, 8 species were common in both animals (Taenia spp., Hymenolepis diminuta, $H$. nana, Ancylostoma spp., A. lumbricoides, Capillaria spp., T. leonina and $T$. vulpis). In total, 17 zoonotic parasite species were identified from the study animals (Table 1).

\subsection{Prevalence of Different Parasite Species in Dogs and Cats}

Among all these parasites, Capillaria spp. had the highest prevalence in dogs (86.67\%) and cats $(90 \%)$ followed by Trichuris vulpis $(83.33 \%$ in dogs, $90 \%$ in cats). Other highly prevalent parasites in dogs were Ascaris lumbricoides and Toxocara canis (prevalence $76.67 \%$ for both). Other parasites, which had high prevalence in

Table 1. List of parasite species found in dogs and cats

\begin{tabular}{lll}
\hline & \multicolumn{2}{c}{ Name of the parasites } \\
\hline Found in dogs only $(\mathbf{n = 2})$ & Found in cats only $(\mathbf{n = 7})$ & Found in both dogs and cats $(\mathbf{n = 8})$ \\
\hline Toxocara canis & Sarcocystis spp. & Taenia spp. \\
Linguatula serrata & Toxoplasma spp. & Hymenolepis diminuta \\
& Diphyllobothrium latum & Hymenolepis nana \\
& Heterophyes spp. & Ancylostoma spp. \\
& Opisthorchis spp. & Ascaris lumbricoides \\
& Gnathostoma spp. & Capillaria spp. \\
& Toxocara cati & Toxascaris leonina \\
& & Trichuris vulpis \\
\hline
\end{tabular}




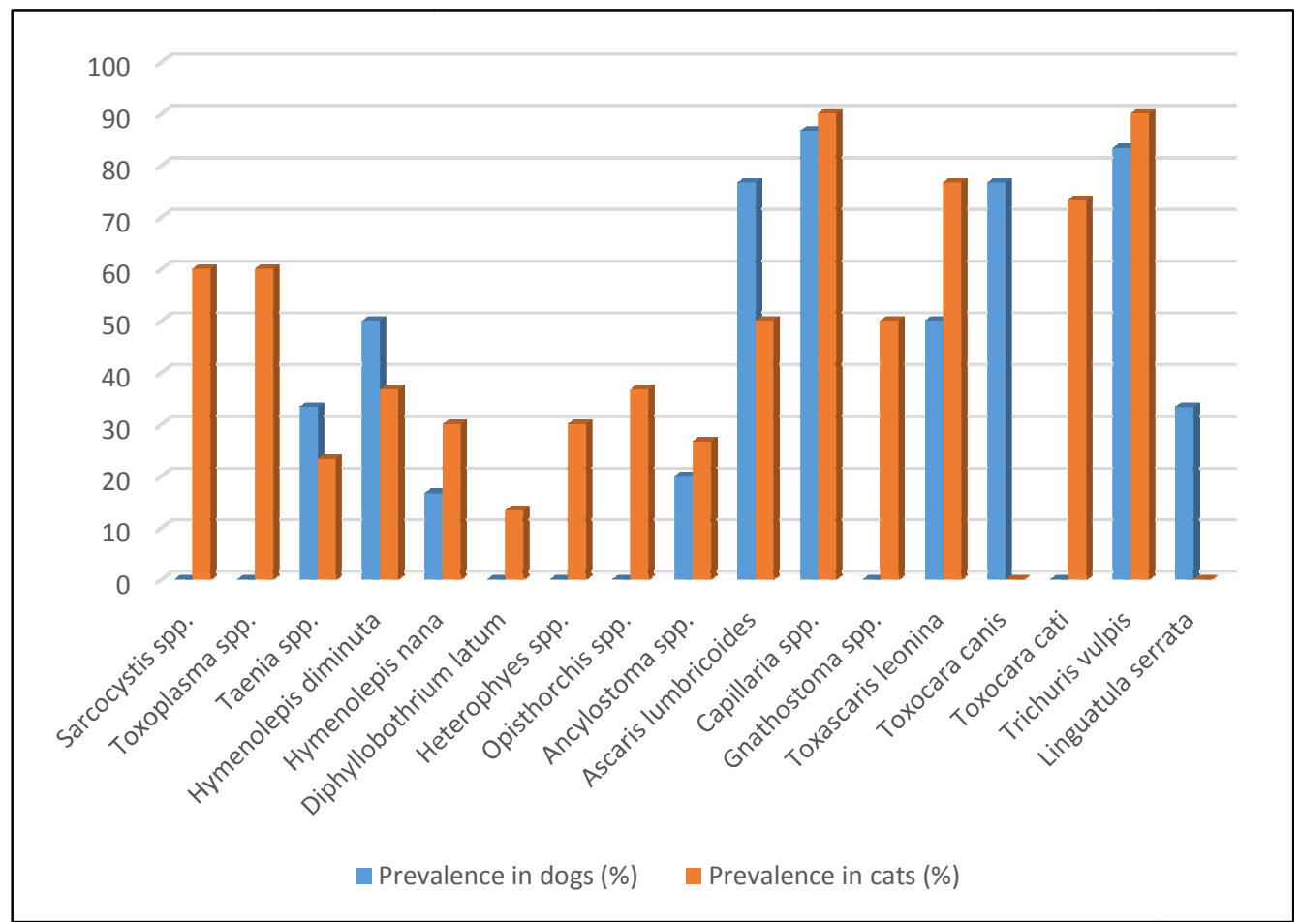

Fig. 2. Prevalence of different parasite species in dogs and cats

cats were - Toxascaris leonina, Toxocara cati, Sarcocystis spp. and Toxoplasma spp. (prevalence $76.67 \%, 73.33 \%, 60 \%$ and $60 \%$, respectively) (Fig. 2).

\subsection{Prevalence of Common Parasite Species Found in Dogs and Cats in Relation to Age}

In this study, among dogs, puppies had the highest parasitic prevalence $100 \%$ followed by young (83.33\%) and adult dogs (81.82\%). In cats, kitten and young had the highest parasitic prevalence $(100 \%)$ followed by adult $(81.82 \%)$.

Among puppies, the highest prevalence was observed for Ascaris lumbricoides, Capillaria spp and Toxocara canis $(100 \%, 85.71 \%$ and $85.71 \%$ respectively). Among young dogs Capillaria spp, Trichuris vulpis, Toxocara canis $(91.67 \%$, $83.33 \%$ and $75 \%$ respectively) and adult dogs, Ascaris lumbricoides, Trichuris vulpis, Capillaria spp $(100 \%, 90.91 \%$ and $81.82 \%$ respectively) had the highest prevalence (Fig. 3).

In kittens, Trichuris vulpis had the highest prevalence (100\%) followed by Gnathostoma spp and Capillaria spp. (85.71\% for both). In young cats, the prevalence of Trichuris vulpis, Capillaria spp, Toxocara cati (100\%, 90.91\%, $75 \%$ respectively) and in adult cats Capillaria spp. (90.91\%), Toxocara cati, Trichuris vulpis (81.82\% in both) was very high (Fig. 4).

\section{DISCUSSION}

The current study aimed to determine the prevalence of infestation of different zoonotic parasite species in dogs and cats from a prominent pet market of the capital city of Dhaka, Bangladesh. Zoonoses or disease caused by human animal contact is becoming increasingly common phenomena in current century. By this study, we were able to identify 17 different parasite species of zoonotic importance in total 60 animals (30 dogs and 30 cats). Apart from 8 common species, 2 more species were exclusively identified in dogs and 7 species in cats. This study suggests that cats in general had more parasite infestation than dogs and more diverse species of parasites were able to infect cats. This has been previously observed in studies in Bangladesh [10] and abroad [4,7,9]. According to the age group of hosts, in both dogs and cats, puppies or kittens along with young age hosts had higher prevalence of parasites compared to adults although the difference was statistically insignificant $(p=.06$ for different age 
group of dogs and $p=.33$ for cats). This has been previously observed in several studies $[13,14]$. This difference in infection according to age of host can happen due to the lower immune function in puppies/kittens and young hosts than adult dogs and cats.

Among all the identified parasites, Capillaria spp. had the highest prevalence in dogs (86.67\%) and cats $(90 \%)$ followed by Trichuris vulpis $(83.33 \%$ in dogs, $90 \%$ in cats). Capillaria spp. is a Trichinelloid nematode commonly known as roundworms causing gastrointestinal infections in both cats and dogs. This parasite can be highly pathogenic to human and can cause severe diarrheal symptoms, even death in certain circumstances [15]. Trichuris vulpis is a whipworm commonly observed in dogs and also in cats [16]. Disease caused by this pathogen is highly prevalent worldwide among people specially children. A study in Thailand found that dogs were acting as common reservoirs for Trichuriasis infection in rural school children [17].

In dogs, high prevalence were also observed for Ascaris lumbricoides and Toxocara canis (76.67\% for both). A. lumbricoides is the most common intestinal Nematode found in children and adults worldwide. This giant roundworm has been a cause of concern globally due to its high prevalence of disease burden in people among Sub Saharan Africa, East Asia and America [18]. $T$. canis is another nematode of zoonotic potential; it can cause Toxocariasis in human specially in children which can affect various body organs of human causing damage in liver and central nervous system in severe cases [19].

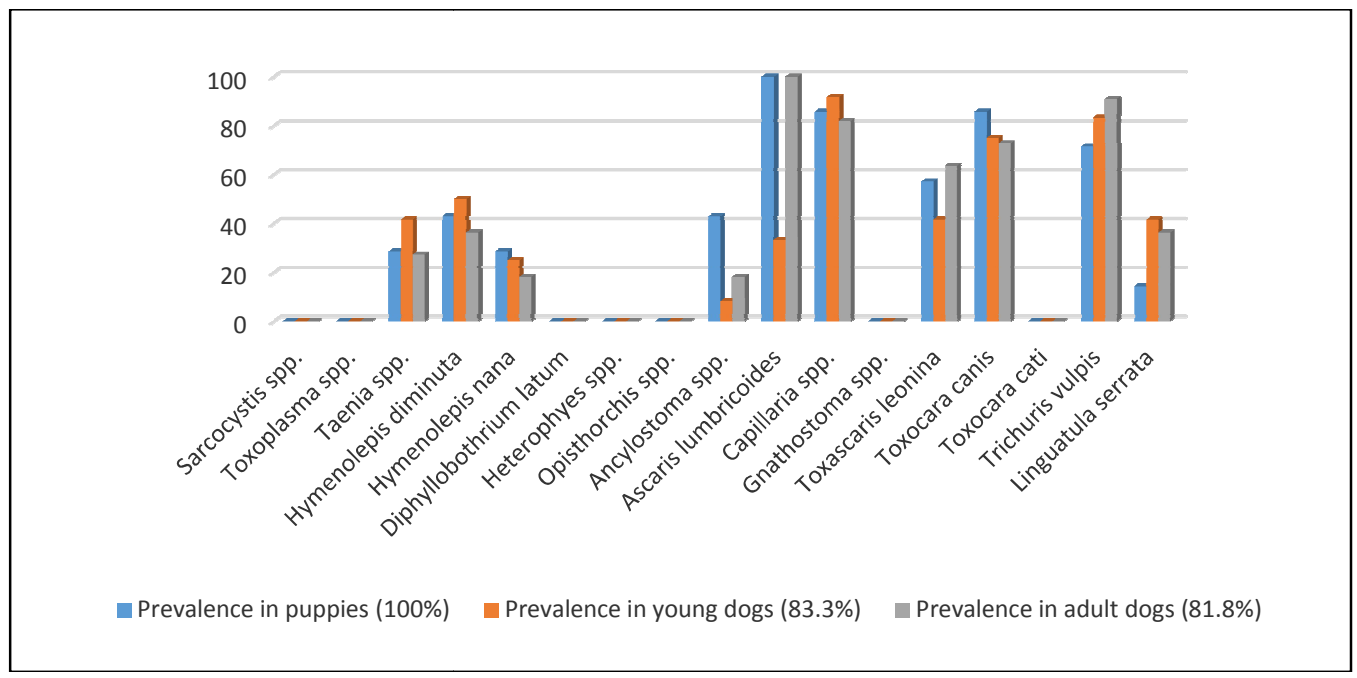

Fig. 3. Age related prevalence of different parasite species in dogs

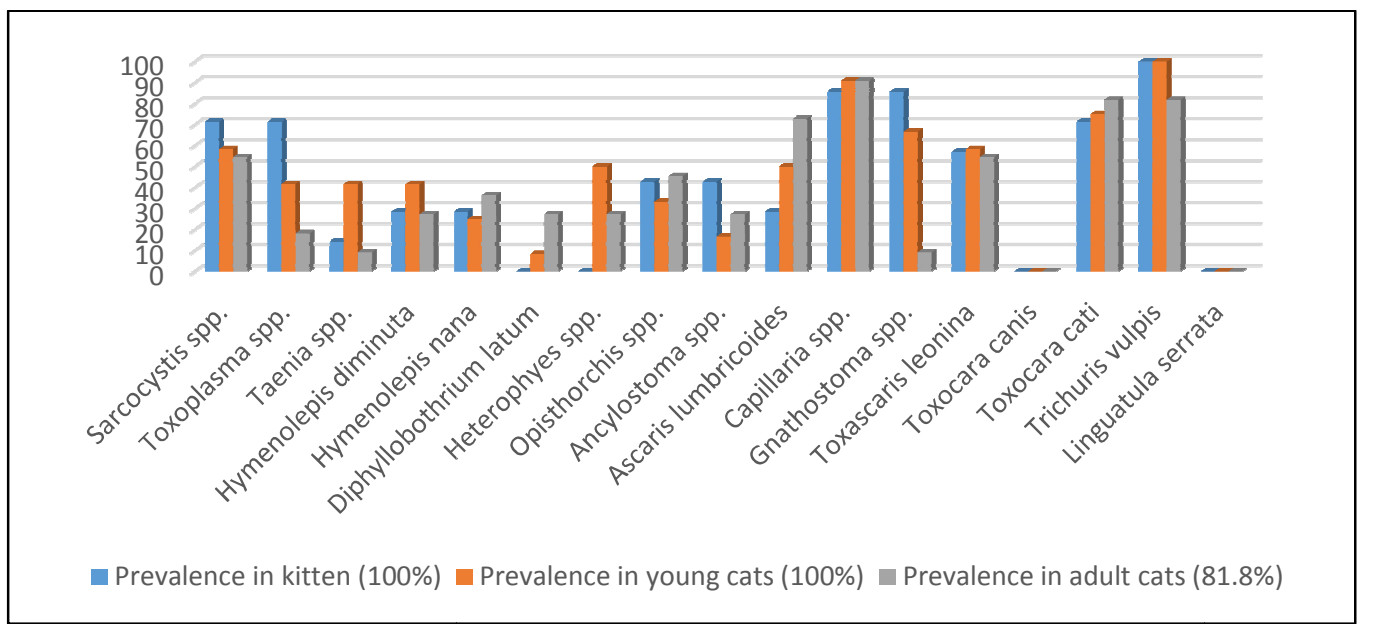

Fig. 4. Age related prevalence of different parasite species in cats 
In cats, high to moderate prevalence was observed for Toxascaris leonina (76.67\%), Toxocara cati (73.33\%), Sarcocystis spp. (60\%) and Toxoplasma (60\%) spp.; all of which can affect human by causing different diseases. $T$. leonina and $T$. cati are highly prevalent in kittens and young cats and can transmit to human by contamination of food with cat feces [14]. On the other hand, Sarcocystis spp. is a zoonotic protozoan which causes diarrhea when oocysts are consumed by human from animals [20]. Toxoplasma spp. is of special concern in households with pregnant women as transplacental transmission of this parasite can cause abnormalities in unborn fetus [21].

The high prevalence of zoonotic parasites observed in the present study is alarming as Katabon pet market is one of the famous pet markets in Dhaka city and has becoming increasingly popular among the pet owners of Bangladesh. Different breeds of dogs and local breed of cats are very popular among urban people as they are easier to handle than other exotic species. The risk of zoonotic infection by these animals can be very high in children and pregnant women as they are immunocompromised and are able to spend more time with household pets. Although the observed animals were kept in separate cages but due to human handling by the pet shop owners/ handlers, they pose a risk of infection among the owners, handlers and buyers.

Moreover, these animals were given cooked food alongside pet food from market which were placed in cages and could have been contaminated easily by the feces of those animals living in the cage. Pets should be handled with proper care and they must be given deworming medicines and vaccines should be regularly administered to avoid further spread of diseases. Proper training should be given to pet handlers when handling the food/ feces to reduce risk of contamination and mass people should be aware about the risk of zoonotic parasite species to avoid potential health hazards.

\section{CONCLUSION}

The current study determines the prevalence of parasites of high zoonotic potential in dogs and cats from a famous pet market in Dhaka, Bangladesh. The findings suggest that parasite burden and risk of zoonotic infection is very high among the city dwellers. Relevant officials should closely monitor the potential health hazard related to zoonotic infection and should take proper steps to create mass awareness about zoonotic threats.

\section{ETHICAL APPROVAL}

As per international standard or university standard written ethical approval has been collected and preserved by the author(s).

\section{COMPETING INTERESTS}

Authors have declared that no competing interests exist.

\section{REFERENCES}

1. Belay ED, et al. Zoonotic disease programs for enhancing global health security. Emerg Infect Dis. 2017;23(13): S65-70.

2. GM B. The history of rabies. 2nd ed. Rabies. Amsterdam: Elsevier Inc; 2007.

3. WHO. Novel Coronavirus (2019-nCoV); 2019.

Available:https://www.who.int/emergencies /diseases/novel-coronavirus-2019

4. Komtangi MC, et al. Prevalence of gastrointestinal helminths of dogs in Dschang, Cameroon. Journal of the Cameroon Academy of Sciences. 2005;5 (1):11-14.

5. Serpell J. The domestic dog: Its evolution, behaviour and interactions with people. $6^{\text {th }}$ ed. Cambridge University Press; 1995.

6. Long JL. Introduced mammals of the world: Their history, distribution, and influence. CSIRO Publishing; 2003.

7. Mateus TL, et al. Multiple zoonotic parasites identified in dog feces collected in Ponte de Lima, Portugal-a potential threat to human health. Int J Environ Res Public Health. 2014;11(9):9050-67.

8. Yang $\mathrm{Y}$, Liang $\mathrm{H}$. Prevalence and risk factors of intestinal parasites in cats from China. Biomed Res Int. 2015;2015: 967238.

9. Baneth G, SMT, Otranto D, Guillot J, Blaga R, Deplazes P, Solano-Gallego L. Major parasitic zoonoses associated with dogs and cats in Europe. Journal of Comparative Pathology. 2016;155(1):S54S74.

10. Samad MA. Public health threat caused by zoonotic diseases in Bangladesh. Bangl. J. Vet. Med. 2011;9(2):95-120. 
11. Hamida Khanum SMARA. Association of dogs (Canis lupus familiaris, Linnaeus, 1753) and risk perception of zoonotic infection for humans. National Journal of Life Sciences. 2018;15:27-32.

12. Cheesbrough M. Medical laboratory manual for tropical countries. $2^{\text {nd }}$ ed. London, Boston, Butterworths: Tropical Health Technology; 1987.

13. Beck AM, Katcher AH. Between pets and people: The importance of animal companionship. Purdue University Press; 1996.

14. Rodan I, Sparkes AH. The cat: Preventive healthcare for cats. Saunders; 2011.

15. Saichua P, Nithikathkul C, Kaewpitoon N. Human intestinal capillariasis in Thailand. World Journal of Gastroenterology: WJG. 2008;14(4):506.

16. Jean $A$, Nemzek PAL, Marissa Wolfe, Robert C Dysko, Daniel D Myers Jr. Biology and diseases of dogs. $3^{\text {rd }}$ ed.; 2015.

17. Areekul $\mathrm{P}$, et al. Trichuris vulpis and $T$. trichiura infections among schoolchildren of a rural community in northwestern Thailand: The possible role of dogs in disease transmission. Asian Biomedicine. 2010;4(1):49-60.

18. Silber SA, et al. Efficacy and safety of a single-dose mebendazole $500 \mathrm{mg}$ chewable, rapidly-disintegrating tablet for ascaris lumbricoides and trichuris trichiura infection treatment in pediatric patients: A double-blind, randomized, placebocontrolled, phase 3 study. Am J Trop Med Hyg. 2017;97(6):1851-1856.

19. Aydenizöz-Özkayhan M, Yağcı B, Erat S. The investigation of toxocara canis eggs in coats of different dog breeds as a potential transmission route in human toxocariasis. Veterinary Parasitology. 2008;152(1-2): 94-100.

20. Fayer R. Sarcocystis spp. in human infections. Clinical Microbiology Reviews. 2004;17(4):894-902.

21. Nash JQ, et al. Risk factors for toxoplasmosis in pregnant women in Kent, United Kingdom. Epidemiol Infect. 2005; 133(3):475-83.

(c) 2020 Barua et al.; This is an Open Access article distributed under the terms of the Creative Commons Attribution License (http://creativecommons.org/licenses/by/4.0), which permits unrestricted use, distribution, and reproduction in any medium, provided the original work is properly cited.

Peer-review history:

The peer review history for this paper can be accessed here: http://www.sdiarticle4.com/review-history/54729 